\title{
Autophagy-deficient breast cancer shows early tumor recurrence and escape from dormancy
}

\author{
Hussein F. Aqbi ${ }^{1}$, Liliya Tyutyunyk-Massey ${ }^{2}$, Rebecca C. Keim ${ }^{1,3}$, Savannah E. \\ Butler $^{1,3}$, Theresa Thekkudan ${ }^{2}$, Supriya Joshi ${ }^{4}$, Timothy M. Smith ${ }^{1}$, Dipankar \\ Bandyopadhyay $^{3,5}$, Michael O. Idowu ${ }^{3,6}$, Harry D. Bear ${ }^{3,7}$, Kyle K. Payne ${ }^{8}$, David A. \\ Gewirtz ${ }^{2,3}$ and Masoud H. Manjili1,3,6,9 \\ ${ }^{1}$ Department of Microbiology and Immunology, Virginia Commonwealth University School of Medicine, Richmond, 23298, VA, USA \\ ${ }^{2}$ Department of Pharmacology and Toxicology, Virginia Commonwealth University School of Medicine, Richmond, 23298, VA, USA \\ ${ }^{3}$ Massey Cancer Center, Virginia Commonwealth University School of Medicine, Richmond, 23298, VA, USA \\ ${ }^{4}$ Department of Human and Molecular Genetics, Virginia Commonwealth University School of Medicine, Richmond, 23298, VA, USA \\ ${ }^{5}$ Department of Biostatistics, Virginia Commonwealth University School of Medicine, Richmond, 23298, VA, USA \\ ${ }^{6}$ Department of Pathology, Virginia Commonwealth University School of Medicine, Richmond, 23298, VA, USA \\ ${ }^{7}$ Department of Surgery, Virginia Commonwealth University School of Medicine, Richmond, 23298, VA, USA \\ ${ }^{8}$ Department of Immunology, Moffitt Cancer Center, Tampa, 33612, FL, USA \\ ${ }^{9}$ VCU Institute of Molecular Medicine, Virginia Commonwealth University School of Medicine, Richmond, 23298, VA, USA \\ Correspondence to: David A. Gewirtz, email: david.gewirtz@vcuhealth.org \\ Masoud H. Manjili, email: masoud.manjili@vcuhealth.org \\ Keywords: breast cancer; autophagy; tumor dormancy; tumor escape and relapse; cancer immunotherapy \\ Received: March 15, $2018 \quad$ Accepted: April 07, $2018 \quad$ Published: April 24, 2018
}

Copyright: Aqbi et al. This is an open-access article distributed under the terms of the Creative Commons Attribution License 3.0 (CC BY $3.0)$, which permits unrestricted use, distribution, and reproduction in any medium, provided the original author and source are credited.

\section{ABSTRACT}

Breast cancer patients who initially respond to cancer therapies often succumb to distant recurrence of the disease. It is not clear why people with the same type of breast cancer respond to treatments differently; some escape from dormancy and relapse earlier than others. In addition, some tumor clones respond to immunotherapy while others do not. We investigated how autophagy plays a role in accelerating or delaying recurrence of neu-overexpressing mouse mammary carcinoma (MMC) following adriamycin (ADR) treatment, and in affecting response to immunotherapy. We explored two strategies: 1) transient blockade of autophagy with chloroquine (CQ), which blocks fusion of autophagosomes and lysosomes during ADR treatment, and 2) permanent inhibition of autophagy by a stable knockdown of ATG5 (ATG5 ${ }^{\mathrm{KD}}$ ), which inhibits the formation of autophagosomes in MMC during and after ADR treatment. We found that while CQ prolonged tumor dormancy, but that stable knockdown of autophagy resulted in early escape from dormancy and recurrence. Interestingly, ATG5 ${ }^{\mathrm{KD}}$ MMC contained an increased frequency of ADR-induced polyploid-like cells and rendered MMC resistant to immunotherapy. On the other hand, a transient blockade of autophagy did not affect the sensitivity of MMC to immunotherapy. Our observations suggest that while chemotherapy-induced autophagy may facilitate tumor relapse, cell-intrinsic autophagy delays tumor relapse, in part, by inhibiting the formation of polyploid-like tumor dormancy.

\section{INTRODUCTION}

Autophagy plays a paradoxical role in the promotion and inhibition of cancer. On the one hand, autophagy has a cancer-promoting role by protecting tumor cells from chemotherapy or providing a source of energy for tumor cells to survive under hypoxic and acidic conditions despite the lack of mature vessels [1]. 
On the other hand, inhibition of autophagy by disruption of Beclin 1 or deletion of ATG5 increases the frequency of spontaneous malignancies [2] or liver tumor [3], respectively. Recently, four different mechanisms have been proposed to describe paradoxical functions of autophagy in cancer, which include cytotoxic, cytostatic, cytoprotective and non-protective autophagy [4]. There are also three major types of autophagy which include micro-autophagy involving the direct engulfment of cytosolic material by lysosomes through invagination, chaperone-mediated autophagy involving HSP70 and the lysosomal membrane associated protein $2 \mathrm{~A}$ (LAMP2A), and macro-autophagy which is a highly conserved pathway involving the formation of autophagosomes, which fuse with lysosomes. To this end, ATG5 is involved in the elongation of autophagosomes to engulf toxic material for degradation. A stable knockdown of ATG5 results in the inhibition of the formation of autophagosomes and progression of macro-autophagy [5]. Chloroquine (CQ), on the other hand, does not have any effects on autophagosomes but it blocks the fusion of autophagosomes and lysosomes, thereby preventing the completion of macro-autophagy. In order to investigate the role of macro-autophagy in tumor dormancy and relapse, we performed a transient inhibition of macroautophagy by means of CQ during chemotherapy, which mainly inhibits chemotherapy-induced autophagy while cell-intrinsic autophagy will be restored after the completion of chemotherapy. We also performed a permanent inhibition of cell-intrinsic macro-autophagy by a stable knockdown of ATG5 in tumor cells. We demonstrated that cell-intrinsic, but not chemotherapyinduced, autophagy can inhibit tumor relapse.

\section{RESULTS}

\section{Adriamycin induces autophagy in MMC}

In order to determine whether ADR induces autophagy and in turn establishes tumor dormancy, MMC cells were treated with ADR in the presence or absence of $\mathrm{CQ}$, a pharmacological agent used to block the final stages of autophagy, specifically the fusion of autophagosomes with lysosomes that is necessary for digestion of the cargo in the autophagosomes (frequently termed "autophagic flux"). CQ blocked this autophagic flux as evidenced by the enhanced accumulation of acidic vesicles (red signals) (Figure 1A, ADR and ADR+CQ). We further monitored degradation of the p62/SQSTM1 protein as a marker of autophagic flux, and LC.3B expression as a marker of autophagosomes formation (since LC3 is a component of the autophagosomes). As shown in Figure 1B, ADR did not induce degradation of p62/SQSTM1 although it elevated LC.3B, suggesting that ADR induces autophagy but fails to drive autophagy to completion and p62/ SQSTM1 degradation.
A transient blockade of autophagy by CQ during ADR treatment delays tumor relapse in vitro but not in vivo

Since CQ is being used to sensitize tumor cells susceptible to chemotherapy [6], we sought to determine whether blockade of autophagy by CQ during ADR treatment affects tumor dormancy and relapse. We showed that the presence of CQ during ADR treatment, in vitro, resulted in prolonging tumor dormancy such that, while ADR treated MMC resumed cell proliferation 6 weeks after the treatment, $\mathrm{ADR}+\mathrm{CQ}$ treated $\mathrm{MMC}$ remained dormant (Figure 2A). In order to confirm tumor cell relapse after 6 weeks, flow cytometry analysis of ADR-treated MMC was performed, and indicated a shift of Ki67- non-proliferating cells to Ki67+ proliferating cells with a greater viability (Figure 2B). In fact, MMC cells remained apoptotic by producing floater dead cells following ADR treatment (Supplementary Figure 1A) which compensated for cell proliferation and maintained tumor dormancy for 3 weeks after the completion of ADR treatment. Follow up studies on floater cells showed they were all apoptotic (Supplementary Figure 1B). A transient blockade of autophagy by CQ did not affect susceptibility of tumor cells to ADR-induced apoptosis (Supplementary Figure 2). On the other hand, a transient blockade of autophagy during ADR chemotherapy, in vivo, did not prolong tumor dormancy in FVBN202 mice (Supplementary Figure 3).

\section{A transient blockade of autophagy by CQ during ADR treatment does not change susceptibility of tumor cell to immunotherapy}

In order to determine whether a transient blockade of autophagy during ADR treatment affects susceptibility of dormant MMC to immunotherapy, dormant MMC were cultured with either IFN- $\gamma$ or MMC-reactive T cells three weeks after treatment with $\mathrm{ADR}$ or $\mathrm{ADR}+\mathrm{CQ}$. As shown in Figure 3, untreated MMC or dormant MMC treated with ADR or ADR $+\mathrm{CQ}$ all remained susceptible to IFN- $\gamma$ treatment or T cells.

\section{A stable knockdown of autophagy reduces susceptibility of MMC to ADR treatment}

CQ only transiently blocks fusion of autophagosomes and lysosomes during ADR treatment such that after removal of $\mathrm{CQ}$, accumulated autophagosomes could eventually be fused with lysosomes to complete autophagy. In order to determine the role of autophagy in tumor dormancy or relapse, we used shRNA for a stable knockdown of ATG5 (ATG5 ${ }^{\mathrm{KD}}$ ) which inhibits formation of autophagosomes in MMC. Scrambled shRNA was used as control (Supplementary Figure 4A). The ATG5 ${ }^{\mathrm{KD}}$ MMC and scrambled control MMC were irradiated to confirm that ATG5 ${ }^{\mathrm{KD}}$ MMC cells were deficient in autophagy, using p62 
and LC.3B as read outs (Supplementary Figure 4B). Tumor cells remained intact for the expression of neu antigen, as well as cell proliferation in vitro and in vivo following knockdown of autophagy (Supplementary Figure 4C-4E). Flow cytometry analysis determined a lower level of viability in MMC compared with ATG5 ${ }^{\mathrm{KD}} \mathrm{MMC}$ following ADR treatment (Figure 4).

\section{A stable knockdown of autophagy results in earlier tumor relapse associated with increased frequency of polyploid-like cells and resistance to immunotherapy}

In order to determine whether a higher viability of ATG5 ${ }^{\mathrm{KD}} \mathrm{MMC}$ following ADR treatment (Figure 4) facilitates an earlier tumor relapse compared with wild type $\mathrm{MMC}$, follow up studies were performed for three weeks after ADR treatment. As shown in Figure 5A, ATG5 ${ }^{\mathrm{KD}}$ MMC survived better than autophagy-competent MMC following ADR treatment showing a significantly higher number of cells by 3 weeks after the treatment. Flow cytometry analysis of tumor cells showed greater levels of apoptosis in wild type MMC compared with $\mathrm{ATG}^{\mathrm{KD}} \mathrm{MMC}$ (Figure 5B, $p<0.001$ ). Interestingly, ATG5 ${ }^{\mathrm{KD}} \mathrm{MMC}$ cells contained a higher number of polyploid-like cells following ADR treatment compared with autophagy-competent MMC (Figure 5B, $p<0.03$ ).

In order to determine the in vivo relevance of our in vitro findings, FVBN202 mice were used. Tumor dormancy was first established by ADR treatment in vitro; FVBN202 mice ( $n=7 /$ group) were then challenged i.v. with one million viable dormant tumor cells. Animals were then sacrificed when they became moribund (lost $10 \%$ weight) as a result of massive lung metastasis. As can be seen in Figure 6A, animals that were challenged with ADR-treated ATG5 ${ }^{\mathrm{KD}}$ MMC developed lung metastasis significantly sooner than those that were challenged with ADR-treated MMC. Hematoxylin/eosin and immunohistochemistry analyses of tumor lesions determined a higher frequency of polyploid-like and Ki67+ tumor cells in animals that were challenged with ADRtreated ATG5 ${ }^{\mathrm{KD}}$ MMC (Figure 6B). Finally, ATG5 ${ }^{\mathrm{KD}} \mathrm{MMC}$ were found to be resistant to $\mathrm{T}$ cell-induced apoptosis compared with autophagy-competent MMC (Figure 7).

\section{DISCUSSION}

Cell-intrinsic autophagy is an ongoing process, which regulates cellular metabolism and homeostasis. Autophagy is also induced by insults such as chemotherapy. Here, we studied a paradoxical role of autophagy in tumor promotion and tumor inhibition by a transient inhibition of autophagy only during chemotherapy or a stable knockdown of autophagy in MMC tumor cells. While the former transiently blocked autophagy and cell-intrinsic autophagy was restored after the completion of chemotherapy, the latter permanently blocked chemotherapy-induced autophagy and cell-intrinsic autophagy. We demonstrated that inhibition of chemotherapy-induced autophagy by CQ did not increase susceptibility of tumor cells to chemotherapy-induced apoptosis. Nevertheless, chemotherapy-induced autophagy appeared to accelerate tumor relapse such that use of CQ during chemotherapy delayed tumor relapse in vitro. Our observation is consistent with other reports showing that increased autophagy in residual breast cancer after neoadjuvant chemotherapy was correlated with increased risk of tumor relapse [7]. A transient blockade of autophagy during chemotherapy of tumor-bearing animals did not affect tumor relapse, perhaps, because tumor inhibitory effects of in vivo chemotherapy was not as effective as in vitro drug treatment. Also, chemotherapy-induced autophagy did not affect the sensitivity of tumor cells to apoptosis induced by IFN- $\gamma$ or tumor-reactive T cells.
A

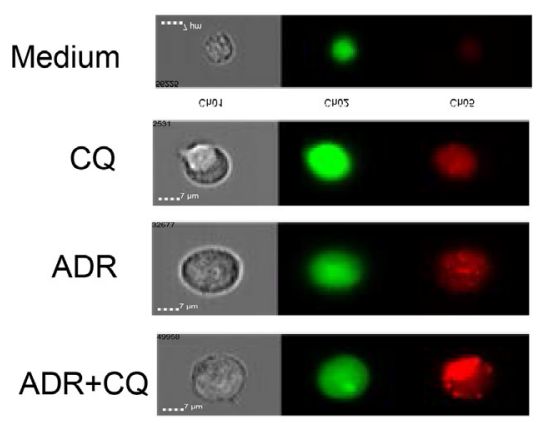

B

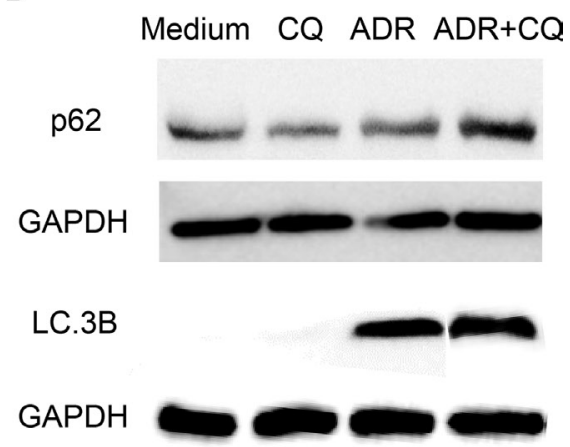

Figure 1: CQ blocks ADR-induced autophagy. MMC tumor cells received three daily doses of ADR alone (1 $\mu$ M ADR for 2 hrs) (ADR) or in the presence of CQ ( $10 \mu \mathrm{M} 3 \mathrm{hrs}$ before ADR and $2 \mathrm{hrs}$ during ADR treatment) (ADR+CQ), washed after each daily treatment and analyzed by acridine orange (AO) one day after the last treatment. Untreated MMC (Medium) or MMC treated with CQ (CQ) served as controls. (A) Acridine orange (AO) staining was analyzed for acidic vesicles (red) using image cytometry. Data represent triplicate experiments. (B) Levels of p62/SQSTM1 and LC.3B after treatment with ADR \pm CQ indicative of autophagy induction in the absence of autophagic flux (B). 
We also demonstrated that, unlike chemotherapyinduced autophagy, cell-intrinsic autophagy accelerated tumor relapse. A stable knockdown of cell-intrinsic autophagy by ATG5 shRNA resulted in a reduced sensitivity of tumor cells to chemotherapy- or $\mathrm{T}$ cellinduced apoptosis, and accelerated tumor relapse in vivo. These effects coincided with an increased frequency of multinuclear polyploid-like dormant cells. These observations suggest that chemotherapy-induced autophagy could have tumor-promoting effects and facilitate tumor relapse, whereas cell-intrinsic autophagy could synergize with cancer therapeutics and delay tumor relapse. In fact, cell-intrinsic autophagy would seem to inhibit the formation of multinuclear cells following chemotherapy, and to prevent chemotherapy-induced genetic instability associated with resistance to cancer therapeutics. Similar observations have been made in other breast tumor models by showing that CQ but not knockdown of Beclin 1 or ATG12 sensitized the tumor to chemotherapy [8]. Therefore, anti-tumor effects of autophagy inhibitors such as CQ is likely to be because of the inhibition of chemotherapy-induced autophagy while anti-tumor effects of autophagy inducers such as rapamycin may result from enhanced cell-intrinsic autophagy $[9,10]$. It has been reported cancer stem cells play a role in tumor dormancy [11] and drug resistance [12], and that immunotherapeutic targeting of breast cancer stem cells inhibits growth of mammary carcinoma [13]. However, we did not detect the enrichment of CD44+CD24- cancer stem cells following ADR-induced tumor dormancy (data not shown).

Anticancer drugs and ionizing radiation tend to induce autophagy in tumor cells [14]. Treatmentinduced autophagy could lead to apoptosis [15] and tumor cell dormancy [16]. We have already reported that dormant tumor cells established by ADR treatment or radiation therapy, in vitro, developed resistance to these treatments but remained susceptible to immunotherapy [17]. Therefore, evaluation of apoptosis or tumor growth inhibition as a single factor without evaluating
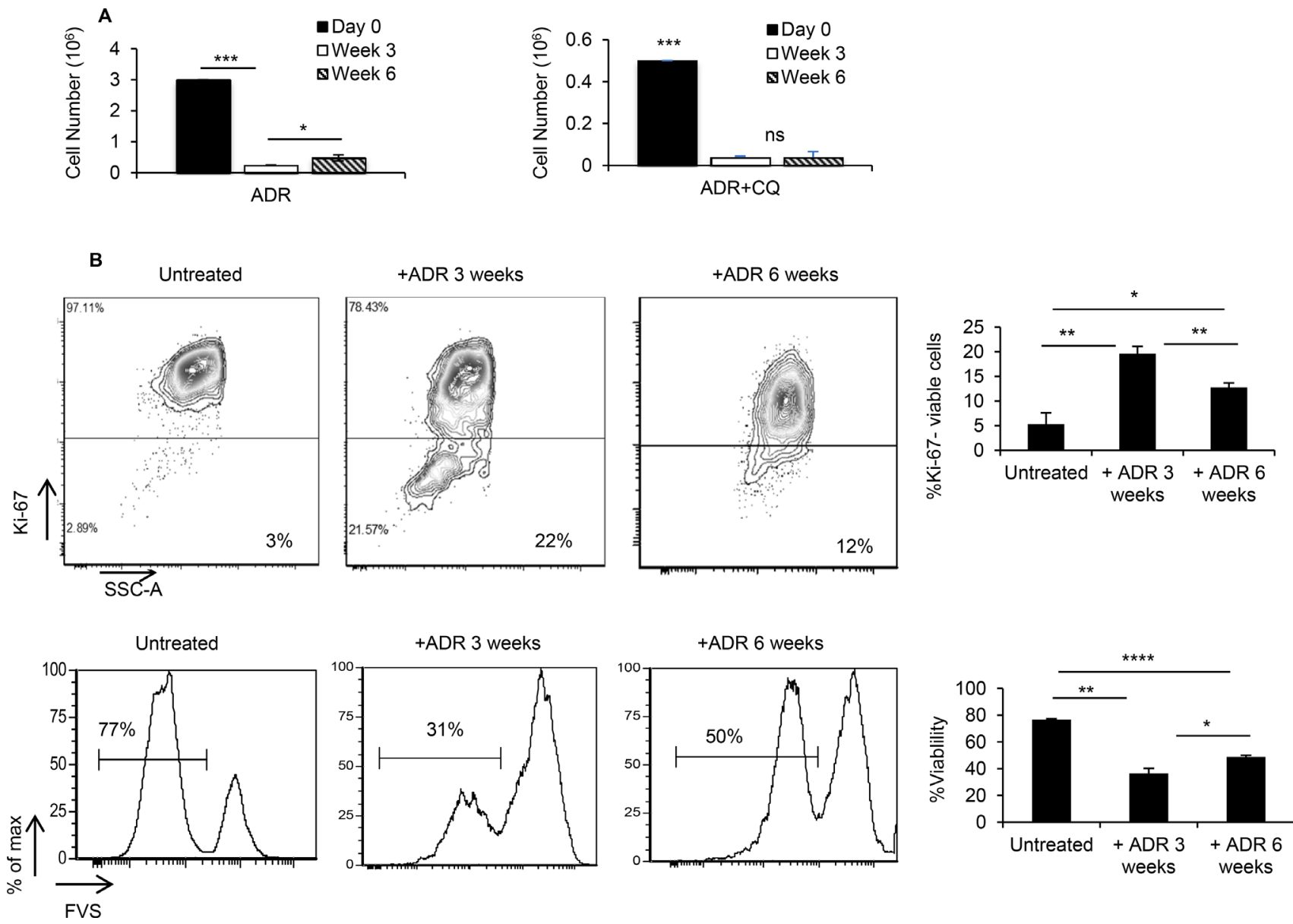

Figure 2: ADR-induced dormant tumor cells remain dormant in the presence of CQ. MMC tumor cells were treated with 3 daily doses of ADR ( $1 \mathrm{uM}$ for $2 \mathrm{hrs}$ ), with one group receiving CQ (10 uM) $3 \mathrm{hrs}$ prior to and during ADR treatment. Both groups remained untreated for 3 weeks and 6 weeks, in vitro. (A) Adherent viable cells were counted using trypan blue exclusion at various time points. Data represent 3 replicates \pm SEM. (B) At weeks 3 and 6 post-treatment, Ki-67 expression (upper panel) and viability (lower panel) were quantified within the population of adherent tumor cells. Data represent 2-3 replicates \pm SEM. Four independent experiments have been carried out which have shown similar results. 


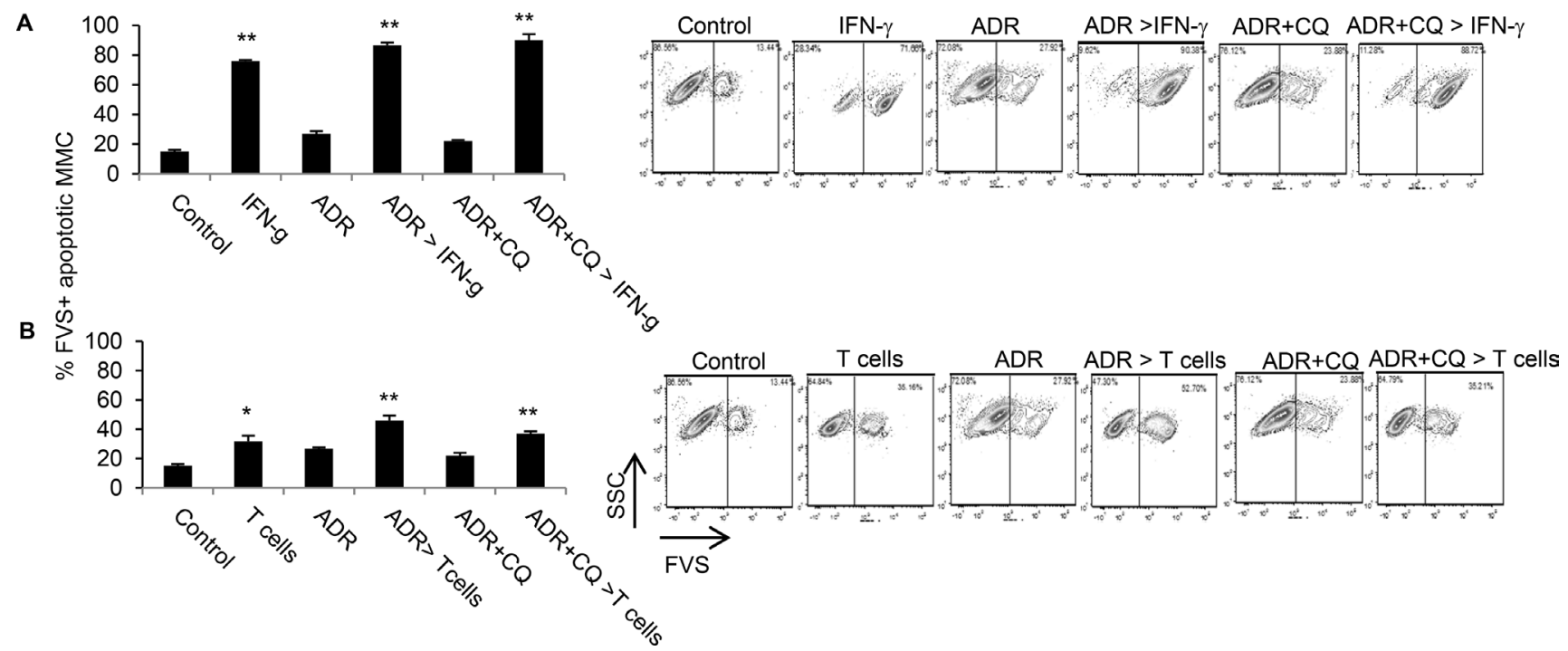

Figure 3: Dormant tumor cells established by ADR or ADR+CQ remain susceptible to immunotherapy. The in vitro tumor dormancy was established three weeks after three daily treatments of MMC with ADR or ADR+CQ. Untreated MMC cells were used as control. (A) Apoptosis was determined by FVS viability staining in MMC (control), ADR-treated dormant MMC (ADR), ADR+CQtreated dormant MMC (ADR+CQ), as well as control MMC cultured with three daily doses of IFN-g and analyzed two days later (50 $\mathrm{ng} / \mathrm{ml}$ ) (IFN-g), ADR-treated dormant MMC cultured with three daily doses of IFN-g $(50 \mathrm{ng} / \mathrm{ml})$ and analyzed two days later (ADR > IFN-g), or ADR+CQ-treated dormant MMC cultured with three daily doses of IFN-g $(50 \mathrm{ng} / \mathrm{ml})$ and analyzed two days later (ADR+CQ $>$ IFN-g). (B) Apoptosis was determined by FVS viability staining of MMC (control), MMC cultured with MMC-sensitized T cells for 48 hrs (T cells), ADR-treated dormant MMC (ADR), ADR-treated dormant MMC cultured with MMC-sensitized T cells for 48 hrs (ADR > T cells), ADR+CQ-treated dormant MMC (ADR+CQ), or ADR+CQ-treated dormant MMC cultured with MMC-sensitized T cells for 48 hrs $(\mathrm{ADR}+\mathrm{CQ}>\mathrm{T}$ cells). Splenic T cells were collected from MMC tumor-bearing FVBN202 mice.
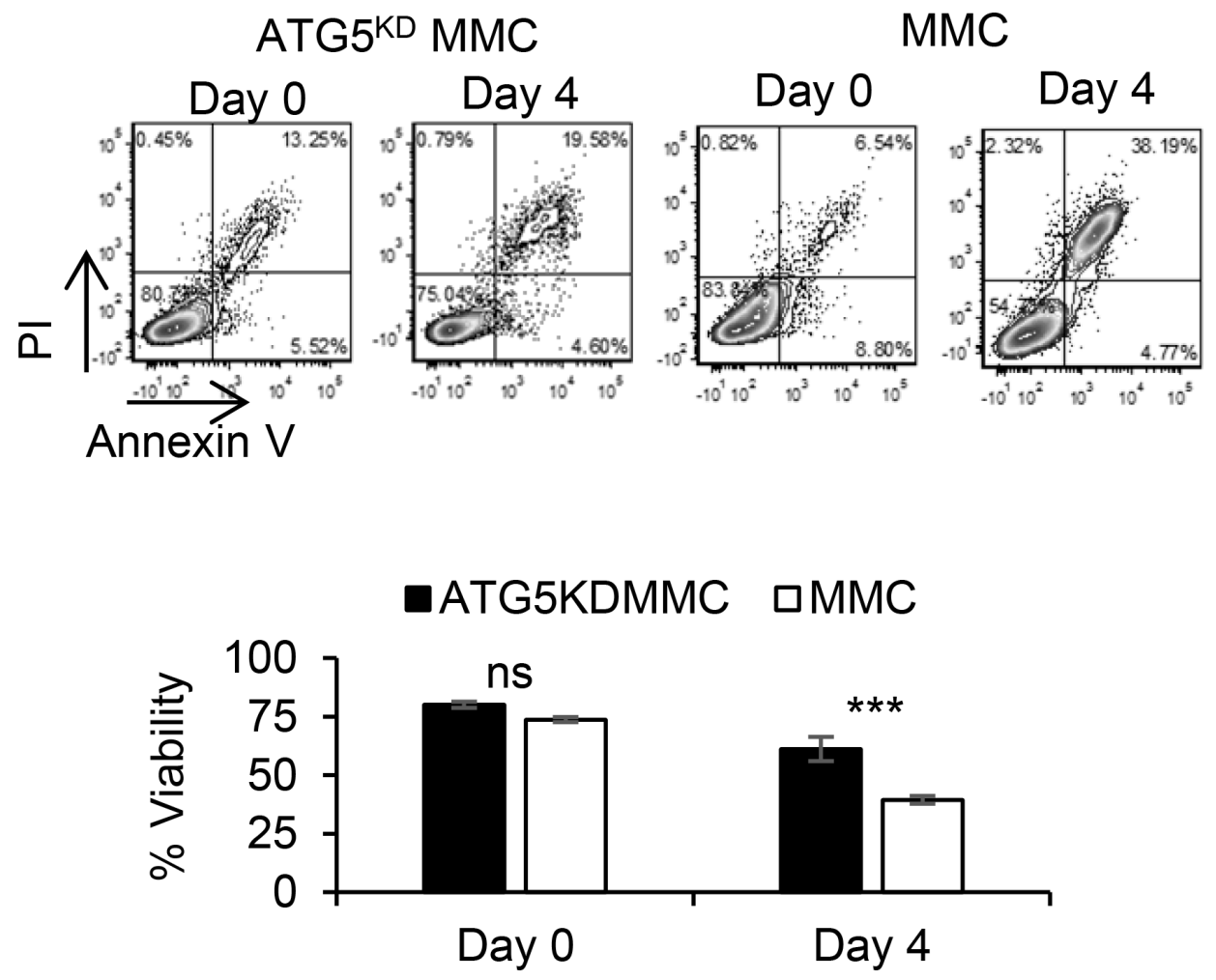

Figure 4: Autophagy knockdown tumor cells become less susceptible to ADR-induced apoptosis. Autophagy-deficient MMC (ATG5 ${ }^{\mathrm{KD}} \mathrm{MMC}$ ) or autophagy-competent MMC (MMC) were treated with a single dose of ADR alone (1 uM ADR for 2 hrs). Tumor cells were analyzed by Annexin V/PI staining prior to treatment (Day 0) or three days after the treatment (Day 4). Experiments were performed in triplicates. 
tumor dormancy and relapse may not be sufficient for understanding anti-cancer efficacy of autophagy inhibitors such as CQ. Inhibition of autophagy by CQ during chemotherapy diminishes the expression of DNA repair proteins, resulting in tumor growth inhibition in carboplatin-resistant BRCA1 wild-type TNBC orthotopic xenografts [18]. In triple negative breast cancer, CQ sensitizes tumor cells to paclitaxel chemotherapy [19]. In several tumor models, CQ synergistically augmented sunitinib cytotoxicity on tumor cells [6]. However, the role of CQ in inhibiting tumor recurrence has yet to be determined.

Cells that are deficient in autophagy show increased levels of reactive oxygen species which result in the accumulation of DNA damage, increased double-strand breaks and polyploid nuclei $[20,21]$. To this end, cell-intrinsic autophagy protects the cell from genomic instability induced by the accumulation of toxins within the cell [22]. It has been reported that Beclin1 knockout mice fail to maintain genomic integrity by increasing DNA double stranded breaks and gene amplifications [20]. A higher expression of Beclin 1 in healthy breast tissue than in breast cancer suggests a deficiency in cell-intrinsic autophagy in tumors [23], which could contribute to genomic instability during tumorigenesis. In breast cancer patients who received adjuvant chemotherapy, presence of tumor cell intrinsic autophagy contributed to reduced risk of tumor relapse [24]. Expression of ATG5 in the tumor specimens is also associated with relapse-free survival in breast cancer patients [25]. In glioma, reduced tumor cell progression and relapse by knockdown of CDGSH iron sulfur domain 2 (CISD2) was associated with the activation of Beclin 1-mediated autophagy [26].

Our observations suggest that any deficiency in tumor cell-intrinsic autophagy could result in a reduced sensitivity of breast cancer to chemotherapy or immunotherapy. Therefore, IHC analysis of tumor biopsies before and after neoadjuvant or adjuvant chemotherapy could determine cell-intrinsic and chemotherapy-induced autophagy, respectively, and in turn might predict the risk of distant recurrence of the diseases accordingly. In future studies, other murine and human breast tumor cell lines as well as other types of carcinoma cells should be used in order to determine whether our findings offer a general mechanism of autophagy-associated tumor dormancy and relapse, or it might be a cancer specific phenomenon.

\section{MATERIALS AND METHODS}

\section{Tumor cell line}

The neu overexpressing mouse mammary carcinoma (MMC) cell line was established from spontaneous mammary tumors harvested from FVBN202 mice [27]. Tumor cells were maintained in RPMI 1640 supplemented with $10 \%$ FBS.

\section{Genetic silencing of ATG5 in MMC}

Mission shRNA bacterial stocks for ATG5 and scrambled Control were purchased from Sigma Aldrich. Lentiviruses were produced in HEK 293TN cells cotransfected using Endo F ectinTM Lenti Transfection Reagent (GeneCopoeia, 1001-01) with a packaging mixture of psPAX2 and pMD2.G constructs (Addgene). Media containing the viruses was used to infect MMC cells; puromycin $(1 \mu \mathrm{g} / \mathrm{ml})$ was used as a selection marker to enrich for infected cells.

\section{Antibodies}

All antibodies were purchase from Biolegend (San Diego, CA, USA) unless otherwise stated. Antibodies were used as instructed by the supplier. Antibodies
A
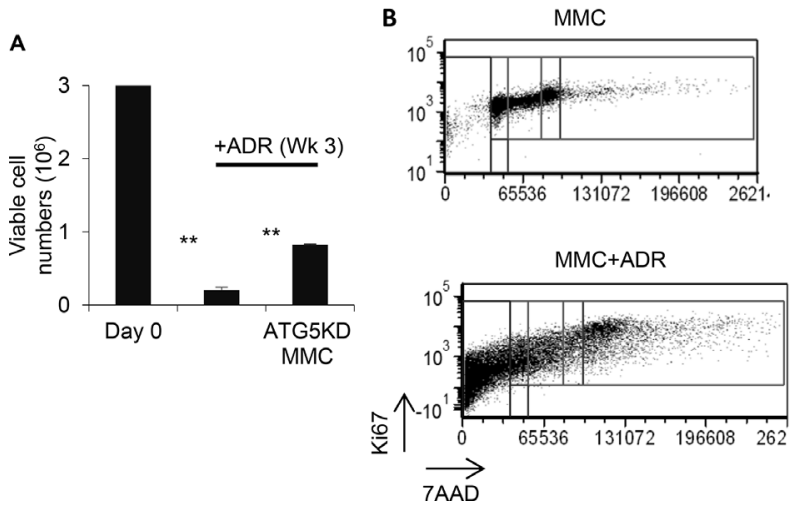
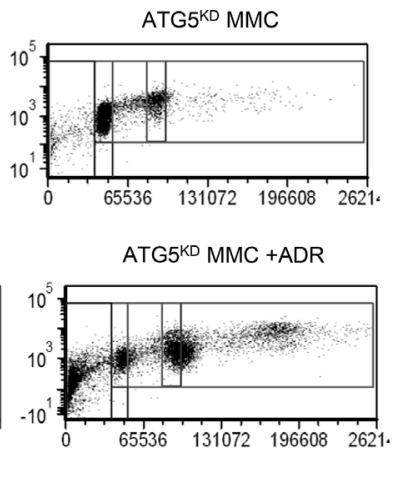

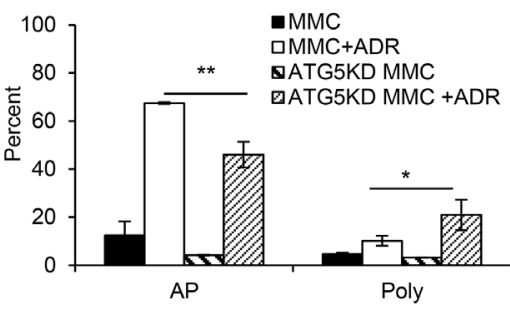

AP

Figure 5: ADR-induced tumor dormancy in autophagy knockdown tumor cells with polyploid-like morphology compared with autophagy competent tumor cells, in vitro. MMC or ATG5 ${ }^{\mathrm{KD}} \mathrm{MMC}$ tumor cells (3 million cells, Day 0 ) were

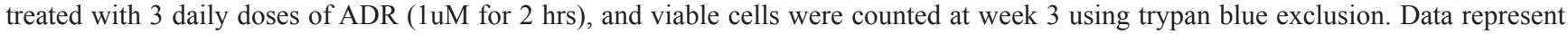
triplicate experiments (A). Dot plots from each experimental group gated for cell cycle phase based upon DNA content (7-AAD) and Ki-67 expression. Events falling to the left of the G1/G0 gates are considered apoptotic cells (AP). Events falling to the far right of the G2/M gate are considered polyploid-like cells (Poly) (B). Three independent experiments have been performed and data represent 3 replicates \pm SEM. 
A
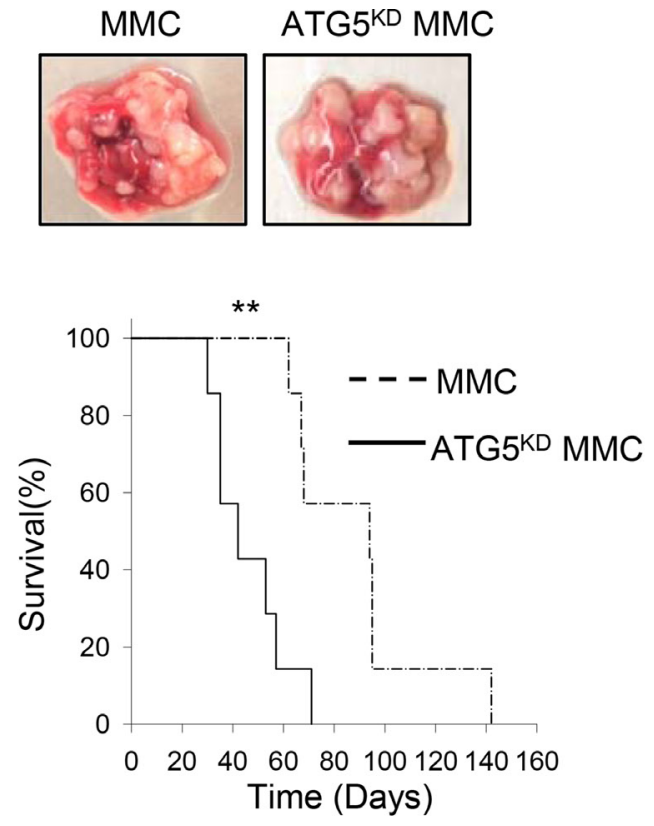

B

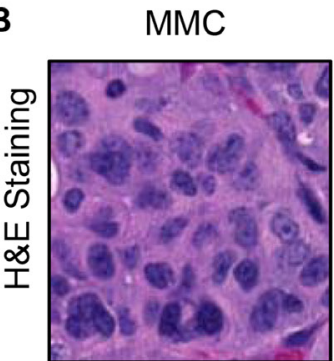

ATG5 ${ }^{\mathrm{KD}} \mathrm{MMC}$
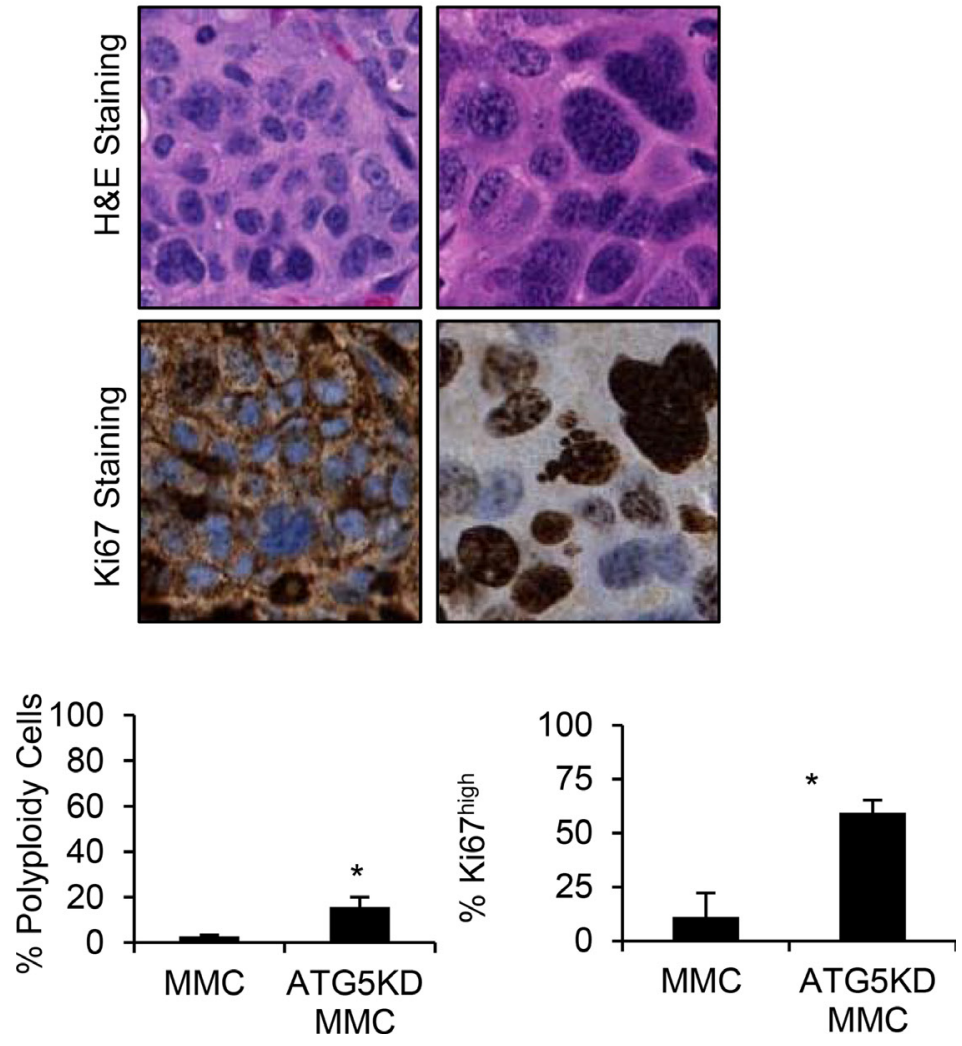

Figure 6: Earlier relapse of autophagy knockdown tumor cells with polyploid morphology compared with autophagy competent tumor cells, in vivo. (A) FVBN202 mice $(n=7)$ were challenged i.v. with $10^{6}$ cells ADR-treated dormant control MMC (MMC), or ADR-treated dormant ATG5 ${ }^{\mathrm{KD}}$ MMC (ATG5 $\left.{ }^{\mathrm{KD}} \mathrm{MMC}\right)$. Animals were euthanized as soon as they became moribund. Representative tumor relapse in the lung and survival curve are shown. (B) Relapsed tumors were collected and immunohistochemistry slides were prepared by either staining samples with hematoxylin and eosin (H\&E) or by Ki67 staining followed by subsequent digitization and analysis with NDP View software (Hamamatsu Photonics). At twenty-times magnification, three representative $0.02 \mathrm{~mm}^{2}$ areas were chosen from the H\&E slides containing approximately 100 cells to measure nuclear envelope size. Cells containing a nuclear envelope equal to or greater than 16 um with visible multi-nuclei were considered polyploid-like or high grade cells. The corresponding cell was then analyzed on the Ki67 stained slide to determine Ki67 expression levels. Data was collected from three biological samples. Significance is based on a two-tailed $t$-test of $p<0.05$. 
include: anti-CD16/32 (clone 93), APC-anti-mouse IgG (Poly4053), PE-Ki67 (16A8), Alexa flour 488-Ki67 (11F6), Brilliant Violet 605-CD45 (30-F11), FITCAnnexin V, APC-Annexin V, 7-AAD viability staining solution and Propidium Iodide solution (PI), mouse anti-rat neu (anti-c-Erb2/c-Neu; 7.16.4, Calbiochem, Billerica, MA, USA), FITC-FVS (BD Biosciences). All reagents were used at the manufacturer's recommended concentration.

\section{Mice}

FVBN202 transgenic female mice (The Jackson Laboratory; Bar Harbor, ME, USA) were used. These mice overexpress non-mutated, non-activated rat neu transgene under the regulation of the mouse mammary tumor virus promoter [28]. These mice develop premalignant mammary hyperplasia similar to ductal carcinoma in situ prior to the development of spontaneous carcinoma [29]. These studies have been reviewed and approved by the Institutional Animal Care and Use Committee at Virginia Commonwealth University.

\section{Experimental tumor dormancy}

In vitro tumor dormancy was established by the treatment of MMC or ATG5 $5^{\mathrm{KD}}$ MMC tumor cells with 3 daily doses of ADR (Sigma-Aldrich, 1uM for $2 \mathrm{hrs).} \mathrm{During} \mathrm{ADR}$ treatment, MMC tumor cells were cultured without or with CQ (Sigma-Aldrich, $10 \mathrm{uM}, 3 \mathrm{hrs}$ prior to and during ADR treatment). By 2 weeks after the treatment, all groups did not show any increases in the number of adherent cells, which is the characteristic of tumor dormancy. For in vivo induction of tumor dormancy, FVBN202 mice were challenged with ADR-treated dormant MMC or ATG5 ${ }^{\mathrm{KD}} \mathrm{MMC}$ (i.v. injection of 1 million viable cells), or untreated MMC followed by 3 weekly treatments of ADR (i.v., $9 \mathrm{mg} / \mathrm{kg}$ ) or with 3 weekly treatment of $\mathrm{ADR}+60 \mathrm{mg} / \mathrm{kg} \mathrm{CQ}$ (i.p.).

\section{Cytotoxicity assay}

Freshly isolated tumor-primed splenic $\mathrm{T}$ cells or ex vivo expanded splenic $\mathrm{T}$ cells were cultured with MMC at a 10:1 E:T ratio in $3 \mathrm{ml}$ complete medium (RPMI-1640 supplemented with $100 \mathrm{U} / \mathrm{ml}$ of penicillin, $100 \mu \mathrm{g} / \mathrm{ml}$ streptomycin, $10 \%$ FBS, $10 \mathrm{mM}$ L-glutamine and $5 \times 10^{-5}$ M 2-mercaptoethanol) with $20 \mathrm{U} / \mathrm{ml}$ of IL-2 (Peprotech) in 6 well culture dishes. After 48 hs cells were harvested and stained for neu (anti-c-Erb2/c-Neu, Calbiochem), Annexin V and PI according to the manufacturer's protocol (BD Pharmingen). Flow cytometry was used to analyze the viability of neu positive cells $[17,30]$.

IFN- $\gamma$ ELISA. Reprogrammed immune cells were cultured in complete medium with irradiated (140 Gy) tumor cells, ADR-treated dormant MMC or ADR+CQtreated dormant $\mathrm{MMC}$ at a 10:1 ratio for $20 \mathrm{hrs}$. Supernatants were then collected and stored at $-80^{\circ} \mathrm{C}$ until assayed. IFN- $\gamma$ was detected using a Mouse IFN- $\gamma$ ELISA kit (BD Biosciences), according to the manufacturer's protocol [30].

\section{Statistical analysis}

Data are summarized as means and standard errors of the mean (SEM) with differences between groups being illustrated with graphical data presented as mean \pm SEM. Statistical comparisons were made using a onetailed or two-tailed Student $t$ test and a $p$-value $<0.05$ was considered significant $\left(^{*}:<0.05,{ }^{* *}:<0.005 .^{* * *}:<0.0005\right.$, $\left.{ }^{* * * *}:<0.00005\right)$.

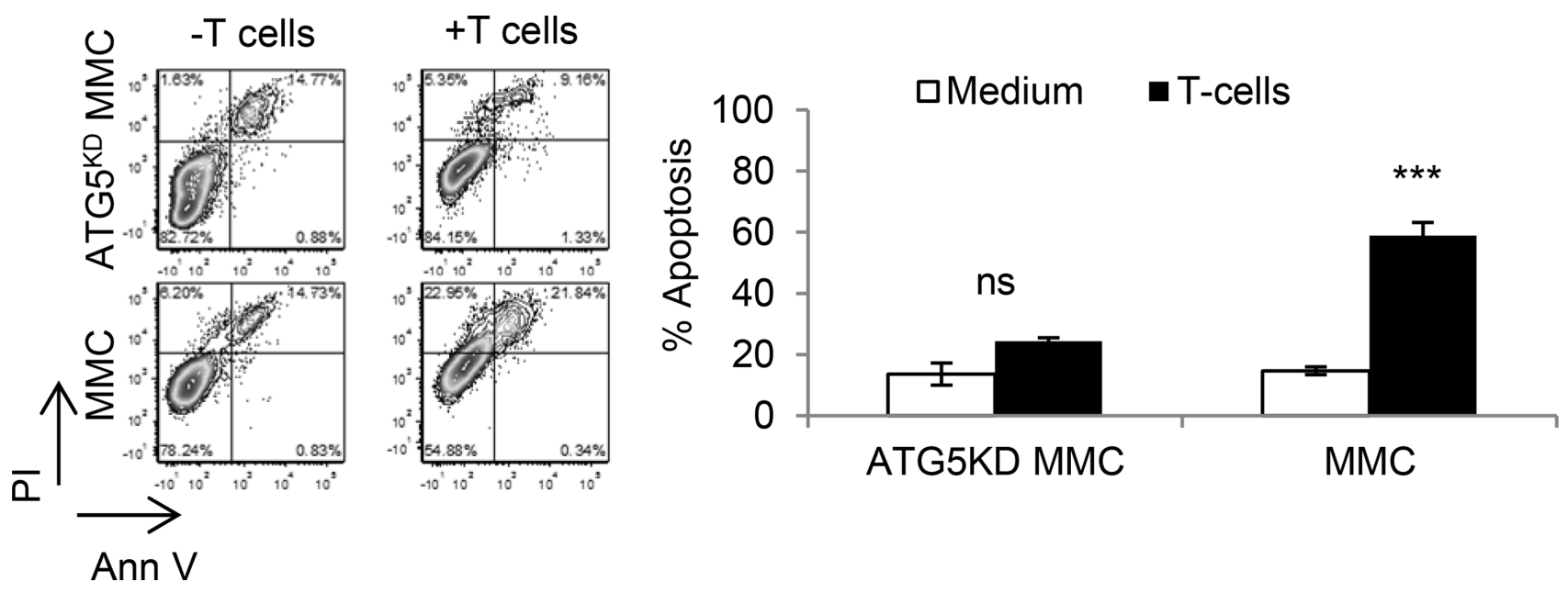

Figure 7: Autophagy knockdown tumor cells become resistant to T cell-induced apoptosis. Neu overexpressing autophagydeficient MMC (ATG5 ${ }^{\mathrm{KD}} \mathrm{MMC}$ ) or autophagy-competent MMC (MMC) were co-cultured with MMC-sensitized T cells and then gated CD45-Neu+ tumor cells were analyzed by Annexin V/PI staining. Data represents triplicate experiments. 


\section{Abbreviations}

ADR, Adriamycin, ATG5, Autophagy-related gene 5, BRCA1, Breast cancer gene 1, CQ, chloroquine, HSP70, Heat shock protein 70 , IHC, Immunohistochemoistry LAMP2A, Lysosomal membrane associated protein $2 \mathrm{~A}$, MMC, neu-overexpressing mouse mammary carcinoma, TNBC, Triple negative breast cancer.

\section{Author contributions}

M.H.M., D.A.G., K.K.P. contributed to the study's conception, design, experimental and analytical performance, and writing of the manuscript. H.F.A., L.T-M., T.T., R.C.K., S.J., S.E.B., T.M.S. contributed to the study's experimental and analytical performance and writing of the manuscript. D.B. contributed to statistical analysis and writing of the manuscript. H.D.B. and M.O.I. contributed to analytical performance and writing of the manuscript.

\section{ACKNOWLEDGMENTS}

We thank Julie Farnsworth for her expertise in image cytometry and immense dedication to furthering the research at our institution. We gratefully acknowledge the support of VCU Massey Cancer Centre and the Commonwealth Foundation for Cancer Research.

\section{CONFLICTS OF INTEREST} disclose.

Authors have no potential conflicts of interest to

\section{FUNDING SUPPORT}

This work was supported by the Office of the Assistant Secretary of Defense for Health Affairs through the Breast Cancer Research Program under Award No. W81XWH-14-1-0087 (MHM) and W81XWH-14-1-0088 (DAG), and a pilot funding from the VCU Massey Cancer Center (MHM). Opinions, interpretations, conclusions, and recommendations are those of the authors and are not necessarily endorsed by the U.S. Department of Defense. Services and products in support of the research project were generated by the VCU Massey Cancer Center Flow Cytometry Shared Resource, supported, in part, with funding from the U.S. National Institutes of HealthNational Cancer Institute Cancer Center Support Grant P30 CA016059.

\section{REFERENCES}

1. Galluzzi L, Pietrocola F, Bravo-San Pedro JM, Amaravadi RK, Baehrecke EH, Cecconi F, Codogno P, Debnath J,
Gewirtz DA, Karantza V, Kimmelman A, Kumar S, Levine $\mathrm{B}$, et al. Autophagy in malignant transformation and cancer progression. The EMBO journal. 2015; 34:856-880.

2. Qu X, Yu J, Bhagat G, Furuya N, Hibshoosh H, Troxel A, Rosen J, Eskelinen EL, Mizushima N, Ohsumi Y, Cattoretti G, Levine B. Promotion of tumorigenesis by heterozygous disruption of the beclin 1 autophagy gene. The Journal of clinical investigation. 2003; 112:1809-1820.

3. Takamura A, Komatsu M, Hara T, Sakamoto A, Kishi C, Waguri S, Eishi Y, Hino O, Tanaka K, Mizushima N. Autophagy-deficient mice develop multiple liver tumors. Genes \& development. 2011; 25:795-800.

4. Gewirtz DA. The four faces of autophagy: implications for cancer therapy. Cancer research. 2014; 74:647-651.

5. Mizushima N, Ohsumi Y, Yoshimori T. Autophagosome formation in mammalian cells. Cell structure and function. 2002; 27:421-429.

6. Abdel-Aziz AK, Shouman S, El-Demerdash E, Elgendy M, Abdel-Naim AB. Chloroquine synergizes sunitinib cytotoxicity via modulating autophagic, apoptotic and angiogenic machineries. Chemico-biological interactions. 2014; 217:28-40.

7. Chen S, Jiang YZ, Huang L, Zhou RJ, Yu KD, Liu Y, Shao $\mathrm{ZM}$. The residual tumor autophagy marker LC3B serves as a prognostic marker in local advanced breast cancer after neoadjuvant chemotherapy. Clinical cancer research. 2013; 19:6853-6862.

8. Maycotte P, Aryal S, Cummings CT, Thorburn J, Morgan MJ, Thorburn A. Chloroquine sensitizes breast cancer cells to chemotherapy independent of autophagy. Autophagy. 2012; 8:200-212.

9. Rangwala R, Leone R, Chang YC, Fecher LA, Schuchter LM, Kramer A, Tan KS, Heitjan DF, Rodgers G, Gallagher M, Piao S, Troxel AB, Evans TL, et al. Phase I trial of hydroxychloroquine with dose-intense temozolomide in patients with advanced solid tumors and melanoma. Autophagy. 2014; 10:1369-1379.

10. Arcella A, Biagioni F, Antonietta Oliva M, Bucci D, Frati A, Esposito V, Cantore G, Giangaspero F, Fornai F. Rapamycin inhibits the growth of glioblastoma. Brain research. 2013; 1495:37-51.

11. Carcereri de Prati A, Butturini E, Rigo A, Oppici E, Rossin M, Boriero D, Mariotto S. Metastatic Breast Cancer Cells Enter Into Dormant State and Express Cancer Stem Cells Phenotype Under Chronic Hypoxia. Journal of cellular biochemistry. 2017; 118:3237-3248.

12. Santisteban M, Reiman JM, Asiedu MK, Behrens MD, Nassar A, Kalli KR, Haluska P, Ingle JN, Hartmann LC, Manjili MH, Radisky DC, Ferrone S, Knutson KL. Immune-induced epithelial to mesenchymal transition in vivo generates breast cancer stem cells. Cancer research. 2009; 69:2887-2895.

13. Cecil DL, Slota M, O’Meara MM, Curtis BC, Gad E, Dang Y, Herendeen D, Rastetter L, Disis ML. Immunization against HIF-1alpha Inhibits the Growth of Basal Mammary 
Tumors and Targets Mammary Stem Cells in vivo. Clinical cancer research. 2017; 23:3396-3404.

14. Zeng X, Kinsella TJ. Impact of Autophagy on Chemotherapy and Radiotherapy Mediated Tumor Cytotoxicity: "To Live or not to Live". Frontiers in oncology. 2011; 1:30.

15. Shen S, Kepp O, Michaud M, Martins I, Minoux H, Metivier D, Maiuri MC, Kroemer RT, Kroemer G. Association and dissociation of autophagy, apoptosis and necrosis by systematic chemical study. Oncogene. 2011; 30:4544-4556.

16. Sosa MS, Bragado $\mathrm{P}$, Debnath J, Aguirre-Ghiso JA. Regulation of tumor cell dormancy by tissue microenvironments and autophagy. Advances in Experimental Medicine and Biology. 2013; 734:73-89.

17. Payne KK, Keim RC, Graham L, Idowu MO, Wan W, Wang XY, Toor AA, Bear HD, Manjili MH. Tumor-reactive immune cells protect against metastatic tumor and induce immunoediting of indolent but not quiescent tumor cells. J Leukoc Biol. 2016; 100:625-35.

18. Liang DH, Choi DS, Ensor JE, Kaipparettu BA, Bass BL, Chang JC. The autophagy inhibitor chloroquine targets cancer stem cells in triple negative breast cancer by inducing mitochondrial damage and impairing DNA break repair. Cancer letters. 2016; 376:249-258.

19. Choi DS, Blanco E, Kim YS, Rodriguez AA, Zhao H, Huang TH, Chen CL, Jin G, Landis MD, Burey LA, Qian W, Granados SM, Dave B, et al. Chloroquine eliminates cancer stem cells through deregulation of Jak2 and DNMT1. Stem cells (Dayton, Ohio). 2014; 32:2309-2323.

20. Karantza-Wadsworth V, Patel S, Kravchuk O, Chen G, Mathew R, Jin S, White E. Autophagy mitigates metabolic stress and genome damage in mammary tumorigenesis. Genes \& development. 2007; 21:1621-1635.

21. Mathew R, Kongara S, Beaudoin B, Karp CM, Bray K, Degenhardt K, Chen G, Jin S, White E. Autophagy suppresses tumor progression by limiting chromosomal instability. Genes \& development. 2007; 21:1367-1381.

22. Mizushima N, Levine B, Cuervo AM, Klionsky DJ. Autophagy fights disease through cellular self-digestion. Nature. 2008; 451:1069-1075.

23. Liang XH, Jackson S, Seaman M, Brown K, Kempkes B, Hibshoosh H, Levine B. Induction of autophagy and inhibition of tumorigenesis by beclin 1. Nature. 1999; 402:672-676.

24. Ladoire S, Penault-Llorca F, Senovilla L, Dalban C, Enot D, Locher C, Prada N, Poirier-Colame V, Chaba K, Arnould L, Ghiringhelli F, Fumoleau P, Spielmann M, et al. Combined evaluation of LC3B puncta and HMGB1 expression predicts residual risk of relapse after adjuvant chemotherapy in breast cancer. Autophagy. 2015; 11:1878-1890.

25. Wang L, Yao L, Zheng YZ, Xu Q, Liu XP, Hu X, Wang $\mathrm{P}$, Shao ZM. Expression of autophagy-related proteins ATG5 and FIP200 predicts favorable disease-free survival in patients with breast cancer. Biochemical and biophysical research communications. 2015; 458:816-822.

26. Sun AG, Meng FG, Wang MG. CISD2 promotes the proliferation of glioma cells via suppressing beclin1mediated autophagy and is targeted by microRNA449a. Molecular medicine reports. 2017; 16:7939-7948.

27. Kmieciak M, Knutson KL, Dumur CI, Manjili MH. HER2/neu antigen loss and relapse of mammary carcinoma are actively induced by $\mathrm{T}$ cell-mediated anti-tumor immune responses. European journal of immunology. 2007; 37: 675-685.

28. Guy CT, Webster MA, Schaller M, Parsons TJ, Cardiff RD, Muller WJ. Expression of the neu protooncogene in the mammary epithelium of transgenic mice induces metastatic disease. Proceedings of the National Academy of Sciences of the United States of America. 1992; 89:10578-10582.

29. Kmieciak M, Morales JK, Morales J, Bolesta E, Grimes M, Manjili MH. Danger signals and nonself entity of tumor antigen are both required for eliciting effective immune responses against HER-2/neu positive mammary carcinoma: implications for vaccine design. Cancer Immunol Immunother. 2008; 57:1391-1398.

30. Kmieciak M, Basu D, Payne KK, Toor A, Yacoub A, Wang XY, Smith L, Bear HD, Manjili MH. Activated NKT cells and NK cells render T cells resistant to myeloid-derived suppressor cells and result in an effective adoptive cellular therapy against breast cancer in the FVBN202 transgenic mouse. J Immunol. 2011; 187:708-717. 